\title{
Spatial structure of genetic variation in a population of the endangered plant Cerastium fischerianum var. molle (Caryophyllaceae)
}

\author{
Masayuki Maki ${ }^{1, *}$ and Tetsukazu Yahara ${ }^{2}$ \\ ${ }^{1}$ Department of Biology, Fukuoka University of Education, \\ Akama, Munakata, Fukuoka 811-41, and \\ ${ }^{2}$ Department of Biology, Faculty of Science, Kyushu University, \\ Hakozaki, Fukuoka 812-81, Japan
}

(Received 26 June 1997, accepted 21 August 1997)

\begin{abstract}
The spatial genetic structure within a population of an endangered plant, Cerastium fischerianum var. molle, was examined using spatial autocorrelation analysis. All individuals in $22 \times 15 \mathrm{~m}$ area in a population were mapped and genotyped by three allozyme loci. Many spatial autocorrelation indices (Moran's $I$ ) were significantly larger than the expected values and their averages across three marker loci examined were positive in the short distance classes and the opposite pattern was shown in the larger distance classes, suggesting that individuals located nearly each other tend to have similar genotypes and pairs of individuals located far from each other tend to have different genotypes. Because the marker loci are considered to be neutral and the linkage disequilibria were not detected, the spatial genetic structure observed in this study seems to result from the restricted gene flow because of predominant selfing of $C$. fischerianum var. molle.
\end{abstract}

\section{INTRODUCTION}

Spatial genetic structure within a population is one of the recent topics in population genetics. In last two decades, many theoretical and experimental studies focused on intrapopulation genetic structure (Heywood, 1991; Epperson, 1993). Although Wright's $F$-statistics has often been used for the measurement of genetic structure within a population, a recent theoretical study revealed that spatial autocorrelation analysis is more powerful than $F$ statistics (Epperson and Li, 1996). Epperson and Li (1996) showed that as much as 10 to 20 times samples are necessary for $F$-statistics to have power similar to autocorrelation measures based on individual genotypes. Furthermore, the experimental studies employing both spatial autocorrelation analysis and $F$-statistics showed that spatial genetic structure was detected by the former, but not by the latter (Bacilier et al., 1994; Alvarez-Buylla et al., 1996).

Spatial autocorrelation analysis has been often employed in plant populations, because plants are sessile and gene flow via pollen or seeds is expected to be highly restricted. Most studies on outcrossing plant populations detected little spatial structure within a population (Heywood, 1991), suggesting that gene flow via pollen (or seed) is sufficiently large to impede population substructuring in outcrossing population. By contrast, a few studies on mixed mating

\footnotetext{
* Corresponding author.
}

populations found distinct intrapopulation genetic structure, suggesting that the genetic patch structures are formed by restricted gene flow within these populations (Schoen and Latta, 1989; Maki and Masuda, 1993). Alternate cause of the micro-scale population structure in the partially selfing species is local hitchhiking selection under high linkage disequilibrium. High linkage disequilibrium has been reported in some predominantly or partially selfing species (Allard et al., 1972; Parker, 1988; Waller and Knight, 1989). Although most marker loci used in spatial autocorrelation analysis are considered to be selectively neutral (Kimura, 1983), it is possible that the loci susceptible to micro-scale environmental variability are closely linked to the marker loci in species under high linkage disequilibrium.

Information on spatial genetic structure within a population is useful for a conservation program of endangered species. A sampling method for seed stocks or an area size necessary for population preservation should be determined based on the population genetic structure. However, few studies concerned spatial genetic structure within a population of an endangered species. Especially, in an endangered plant species which is predominantly selfing, it is essential to measure intrapopulation genetic structures, because the species is expected to have distinct spatial structures within a population. In this study, we report spatial autocorrelation of genotypes in a population of an endangered and predominantly selfing species Cerastium 
fischerianum var. molle.

\section{MATERIALS AND METHODS}

Plant materials. C. fischerianum var. molle Ohwi (Caryophyllaceae) is a perennial herb occurring in rocky sites or grasslands near seashore. This species is distributed in northern area of Kyushu including some islands and southernmost part of Korea (Miki, 1982). Nakanishi (1992) reported nine populations in Nagasaki and Fukuoka Prefecture in Japan, and Kawanari and Kurashige (1993) found five additional populations in Saga Prefecture. Red Data Book of Japanese wild plants (EPSG, 1989) listed $C$. fischerianum var. molle as an 'endangered species' and pointed out that its habitat is getting worse because of garbage dashed up on the shore and water pollu-tion. This species flowers in the late spring to early summer, and disperses seeds in a few weeks after flowering by the ballistic mode (Nakanishi, 1992). Because vegetative reproduction is highly limited, recognition of a individual plant is relatively easy. The selfing rate of the species is more than 0.9 in all populations examined preliminarily (M. Maki, unpublished data).

Electrophoresis. In June of 1997, leaves from 170 flowering individual were collected within $22 \times 15 \mathrm{~m}$ area in a population in Ikitsuki Isl. of Nagasaki Prefecture, Japan. The reason why we chose this population is that it has more allozyme variation than the others most of which have no or one polymorphic locus (M. Maki, unpublished data). Sample leaves kept on ice were transported to the laboratory within a day. The samples were refrigerated for 1-4 days until electrophoresis were carried out. Three polymorphic loci (Pgi-2, glucose-6-phosphate isomerase; 6pgdh4, 6-phosphogluconate dehydrogenase; $S k d h$, shikimate dehydrogenase) were resolved and stained by the standard polyacrylamide gel electrophoresis following Tsumura et al. (1990). Genotypes of these polymorphic loci, Pgi-2, $6 p g d h-4$, and $S k d h$, were determined for 170,151 , and 168 individuals, respectively.

Wright's inbreeding coefficient $(f)$. Wright's inbreeding coefficient $(f)$ (Wright, 1951) was estimated for each loci as $f=1-n_{o} / n_{e}$, where $n_{o}$ and $n_{e}$ are the observed number of heterozygotes and the expected number of heterozygotes, respectively. The significance of $f$ was examined by computing $\chi^{2}$ value for each locus (Li and Horvitz, 1953).

Spatial autocorrelation statistics. The genotypic data were coded such that allele frequency values of $0.0,0.5$, or 1.0 were assigned to individual plants for one allele of each locus. Moran's $I$ values (Sokal and Oden, 1978) were calculated using the SAAP program (ver 4.3) written by D. Wartenberg (1989) for 10 interplant distance classes; $<2.3$ $\mathrm{m},<3.6 \mathrm{~m},<4.8 \mathrm{~m},<5.8 \mathrm{~m},<6.8 \mathrm{~m},<7.9 \mathrm{~m},<8.9 \mathrm{~m},<10.0 \mathrm{~m}$, and $>10.0 \mathrm{~m}$. These interplant distance classes were set up such that each distance class contains equal number of pairs of individual plants. Whether or not each $I$ value deviated significantly from the expected value $E(I)=(N-$ $1)^{-1}$, where $N$ is the number of individuals, was also tested by SAAP. The $I$ values significantly greater than $E(I)$ indicate that similar gene frequencies tend to be shared among neighbors more often than expected at that distance class, and significantly smaller values of $I$ indicate that neighbors are more likely to have different gene frequencies than expected. Overall significance of individual correlograms, which indicate overall spatial autocorrelation across the distance classes was tested using Bonferroni's criteria (Sakai and Oden, 1983).

Estimation of linkage disequilibrium. To estimate the extent of multilocus associations, digenic linkage disequilibria were determined with the composite disequilibrium measure $\Delta_{\mathrm{AB}}$ of Weir and Cockerham (1989) and its normalized value (Lewontin, 1964). The composite disequilibrium measures were calculated in three pairwise combinations of the three marker loci, and their significance was tested by $\chi^{2}$-tests. Because Weir and Cockerham's method does not assume random mating, it is suitable for this study.

\section{RESULTS}

Table 1 shows the allele frequencies and Wright's inbreeding coefficients $(f)$ at the three loci. Because the frequency of $S k d h-c$ is so low that the allele was excluded from the spatial autocorrelation analysis. Thus, Moran's I coefficients were calculated across ten distance classes for an allele in each locus. All inbreeding coefficients were positive and significantly deviated from zero (Table 1).

Table 2 shows the Moran's $I$ at each distance class for each locus. At the shortest distance class $(<2.3 \mathrm{~m})$, the Moran's $I$ is significantly positive for all three loci and the average $I$ value was positive, indicating high genetic similarity was shared among individuals within the distance. At the second to the fourth shortest distance class (2.3$5.8 \mathrm{~m}$ ), four of the nine Moran's $I$ values were significantly positive, indicating also that similar gene frequencies tend

Table 1. Allele frequencies and inbreeding coefficients $(f)$ at the three loci examined

\begin{tabular}{lcccc}
\hline \hline locus & allele & frequency & $f$ & $\left(\chi^{2}\right)$ \\
\hline Pgi-3 & a & 0.211 & & \\
& b & 0.789 & 0.770 & $(100.8)^{*}$ \\
Skdh & a & 0.646 & & \\
& b & 0.334 & & \\
& c & 0.020 & 0.876 & $(118.2)^{*}$ \\
Fpgdh-4 & a & 0.699 & & \\
& b & 0.301 & 0.731 & $(89.8)^{*}$ \\
\hline
\end{tabular}

* Significance at $0.1 \%$ level. 
Table 2. Spatial autocorrelation coefficients (Moran's I) at ten distance classes in a population of Cerastium fisherianum var. molle

\begin{tabular}{llllllllllll}
\hline \hline & 1 & 2 & 3 & 4 & 5 & 6 & 7 & 8 & 9 & 10 & Correlogram \\
\hline Pgi-3 & $0.040^{*}$ & 0.026 & $0.034^{*}$ & $0.040^{*}$ & -0.024 & 0.018 & -0.039 & $-0.061^{*}$ & $-0.052^{*}$ & $-0.042^{*}$ & $\mathrm{P}<0.13$ \\
Skdh & $0.015^{* *}$ & 0.030 & 0.016 & 0.015 & $-0.113^{* *}$ & -0.025 & -0.033 & 0.001 & -0.055 & -0.021 & $\mathrm{P}<0.001$ \\
6pgdh -4 & $0.097^{* *}$ & $0.125^{* *}$ & $0.098^{* *}$ & -0.004 & 0.032 & $-0.050^{*}$ & -0.017 & -0.015 & $-0.102^{* *}$ & $-0.219^{*}$ & $\mathrm{P}<0.001$ \\
Average & 0.051 & 0.060 & 0.049 & 0.030 & -0.035 & -0.019 & -0.030 & -0.025 & -0.070 & -0.094 & \\
\hline
\end{tabular}

Table 3. Estimates of the composite measure of linkage disequilibrium $\left(\Delta_{A B}\right)$ and its normalized value $\left(\Delta_{A B}{ }^{\prime}\right)$ between pairs of three loci examined

\begin{tabular}{lrrc}
\hline \hline \multicolumn{1}{c}{ Loci } & \multicolumn{1}{c}{$\Delta_{A B}$} & \multicolumn{1}{c}{$\Delta_{A B}{ }^{\prime}$} & $\chi^{2}$ \\
\hline Pgi-3/Skdh & 0.012 & 0.209 & $0.220^{\mathrm{ns}}$ \\
Pgi-3/6pgdh-4 & -0.009 & -0.070 & $0.154^{\mathrm{ns}}$ \\
Skdh/6pgdh-4 & 0.025 & 0.128 & $0.661^{\mathrm{ns}}$ \\
\hline
\end{tabular}

${ }^{\mathrm{ns}}$ Not significant.

to be shared among the individuals in these distance classes. By contrast, more than the fourth distance classes $(>5.8$ $\mathrm{m})$, the average Moran's $I$ were negative and eight of $18 \mathrm{I}$ values are significantly negative, indicating that the genotypes more than $6.8 \mathrm{~m}$ far from each other are more likely to have different gene frequencies than expected from random distribution. The overall correlogram was statistically significant for $S k d h$ and $6 p g d h-4$, but not for Pgi-3.

Digenic linkage disequilibria were calculated for pairwise comparisons among the three loci (Table 3). There were no statistically significant deviations from equilibria for any of the comparisons.

\section{DISCUSSION}

The inbreeding coefficients were significantly positive, supporting that C. fischerianum var. molle is predominantly selfing. On the other hand, the digenic linkage disequilibria were not observed in the population examined in this study. A preliminary study on the mating system of C. fischerianum var. molle shows that the selfing rate of the species was more than 0.9 in five populations examined (M. Maki, unpublished data), suggesting that very slight outcrossing $(<10 \%)$ is sufficient to hamper the associations between the loci. Hence local hitchhiking selection is an implausible cause of the spatial genetic structure observed in this study.

As in a few previous studies examining the spatial autocorrelation in plant species with mixed mating system (Schoen and Latta, 1989; Maki and Masuda, 1993), genetic similarity within short distance classes is obvious in this study. In C. fischerianum var. molle, gene flow mainly depends on seed dispersal because the selfing rate is high and sporadic pollen flow may be mediated by near-neighbor movements of opportunistic insects. Although the dominate pollinator has not been identified, small insect species with less flight ability are possible. Because seeds of $C$. fischerianum var. molle have not specialized mechanisms for dispersal (Nakanishi, 1992), they are expected to fall near the mother plants. Therefore, gene flow within a population is highly restricted.

Crawford (1984) discussed the neighborhood area (Wright, 1946) in plant populations, and showed that the neighborhood area $(A)$ is $4 \pi\left(t \sigma_{p}{ }^{2} / 2+\sigma_{s}^{2}\right)$ in mixed selfing plant populations, where $\sigma_{p}, \sigma_{s}$, and $t$ are the variance of the parent-offspring dispersal distribution by pollen, by seeds, and the outcrossing rate, respectively. In a complete selfing population, $A$ becomes $4 \pi \sigma_{s}^{2}$, leading smaller intrapopulation genetic structure than an outcrossing or a mixed mating population. Because, as mentioned above, seed dispersal seems to be highly restricted in $C$. fischerianum var. molle and the species is predominantly selfing, this is the case for the distinct genetic structure in the population.

From the viewpoint of the conservation genetics, we conclude that a sampling method for seed stocks should be taken care in predominantly selfing species. To extract the genetic diversity across entire population efficiently, the sampling should be performed at some meters intervals larger than the genetic patch diameter, because individuals within the patch tend to have similar genotypes. Although caution with sampling interpopulation genetic variation have been exercised (Karron, 1991), similar caution is also necessary for intrapopulation genetic variation in a predominantly selfing plant species.

We thank S. Oiki and S. Horie for the field study. This study was partially supported by the Global Environment Research Fund (F-1) of the Japan Environment Agency.

\section{REFERENCES}

Allard, R. W., Babbel, G. R., Clegg, M. T., and Kahler, A. L. (1972) Evidence for coadaptation in Avena barbata. Proc. Natl. Acad. Sci. USA 69, 3043-3048.

Alvarez-Buylla, E. R., Chaos, A., Piñero, D., and Garay, A. A. (1996) Demographic genetic of a pioneer tree species: patch dynamics, seed dispersal, and seed banks. Evolution 50, 1155-1166.

Bacilieri, R., Labbe, T., and Kremer, A. (1994) Intraspecific genetic structure in a mixed population of Quercus petraea (Matt.) Leible and Q. robur L. Heredity 73, 130-141.

Crawford, T. J. (1984) What is a population? In: Evolutionary Ecology (ed.: E. Shorrocks), pp. 135-173. Blackwell, Oxford.

Epperson, B. K. (1993) Recent advances in correlation studies of spatial patterns of genetic variation. Evol. Biol. 27, 95-155. 
Epperson, B. K. and Li, T. (1996) Measurement of genetic structure within populations using Moran's spatial autocorrelation statistics. Proc. Natl. Acad. Sci. USA 93, 10528-10532.

EPSG (Endangered Plant Survey Group) (1989) The Red Data Book of Japanese Vascular Plants. Nature Conservation Society of Japan, Tokyo. (in Japanese).

Heywood, J. S. (1991) Spatial analysis of genetic variation in plant populations. Annu. Rev. Ecol. Syst. 22, 335-355.

Karron, J. D. (1991) Patterns of genetic variation and breeding systems in rare plant species. In: Genetics and Conservation of Rare Plants (eds.: D. A. Falk and K. E. Holsinger), pp. 8798. Oxford Univ. Press, New York.

Kawanami, M. and Kurashige, Y. (1993) A plant species newly discovered in Saga Prefecture: Cerastium fischerianum var. molle. Saga-no-Shokubutu 50, 43-51. (in Japanese).

Kimura, M. (1983) The Neutral Theory of Molecular Evolution. Cambridge Univ. press, Cambridge.

Lewontin, R. C. (1964) The interaction of selection and linkage. I. General considerations; heterotic models. Genetics 49, 4967.

Li, C. C. and Horvitz, D. G. (1953) Some methods of estimating the inbreeding coefficient. Am. J. Hum. Genet. 5, 107-117.

Maki, M. and Masuda, M. (1993) Spatial autocorrelation of genotypes in a gynodioecious population of Chionographis japonica var. kurohimensis (Liliaceae). Int. J. Plant Sci. 154, 467-472.

Miki, E. (1982) On Cerastium fischerianum Sér. var. molle Ohwi. Acta Phytotaxon. Geobot. 33, 260-263.

Nakanishi, H. (1992) Distribution and ecology of Cerastium fischerianum Sér. var. molle Ohwi. J. Jhytotaxon. Geobot. 40, 29-32. (in Japanese).

Parker, M. A. (1988) Disequilibrium between disease-resistance variants and allozyme loci in a annual legume. Evolution 42, 239-247.

Sakai, A. K. and Oden, N. L. (1983) Spatial pattern of sex expression in silver maple (Acer saccharinum L.): Morisita's index and spatial autocorrelation. Am. Nat. 122, 489-508.

Schoen, D. J. and Latta, R. G. (1989) Spatial autocorrelation of genotypes in populations of Impatiens pallida and Impatiens capensis. Heredity 63 181-190.

Sokal, R. R. and Oden, N. L. (1978) Spatial autocorrelation in biology. I. Methodology. Biol. J. Linn. Soc. 10, 1349-1368.

Tsumura, Y., Tomaru, N., Suyama, N., Na'eim, M., and Ohba, K. (1990) Laboratory manual of isozyme analysis. Bull. Tsukuba Univ. For. 6, 63-95. (in Japanese).

Waller, D. M. and Knight, S. E. (1989) Genetic consequences of outcrossing in the cleistogamous annual, Impatiens capensis. III. Multilocus associations. Heredity 63, 1-9.

Wartenberg, D. (1989) SAAP ver 4.3. Exeter, Setauket.

Weir, B. S. and Cockerham, C. C. (1989) Complete characterization of disequilibrium at two loci. In: Mathematical Evolutionary Theory (ed.: M. W. Feldman), pp. 86-110. Princeton Univ. Press, Princeton.

Wright, S. (1946) Isolation by distance under diverse systems of mating. Genetics 31, 39-59.

Wright, S. (1951) The genetical structure of populations. Ann. Eugen. 15, 323-354. 